\title{
Fortpflanzung und Entwicklung von Anemonia sulcata (Anthozoa, Actiniaria) II. Frühentwicklung, Blastula und Gastrula
}

\author{
W. Schäfer \\ Institut für Zoologie (Lehrstuhl I) der Universität Erlangen-Nürnberg; \\ Universitätsstraße 19, D-8520 Erlangen, Bundesrepublik Deutschland
}

\begin{abstract}
Reproduction and development of Anemonia sulcata (Anthozoa, Actiniaria). II. Early development, blastula and gastrula. Early developmental stages of the Anemonia germ are characterized by asynchronously dividing nuclei and an extreme delay of blastomere differentiation. The nuclei migrate to the periphery, whereas nutritive substances remain in the interior. Following this stage, the appearance of cell boundaries results in the formation of the blastoderm and the simultaneous division of the yolk into many fragments. Most of them are exclusively filled with reserve material; only very few contain zooxanthellae or nuclei. On the embryo's surface, conically shaped bundles of long microvilli are obvious. They appear to be less regularly arranged than the spines of oocytes before insemination. Pigment granules that have originated from fusing Golgi vesicles are crowded peripherally in the blastoderm cells. In the nucleoplasm single annulate lamellae that seem to be cut off from the nuclear envelope can frequently be found. There is no further cellular differentiation until gastrulation is completed. Though yolk-containing cellular fragments occupy nearly the whole blastocoel, entoderm formation occurs by invagination. Ultrastructural observations provide evidence of the existence of interstices between entoderm cells that allow all nutritive substances to pass gradually into the gastric cavity. In the region of the blastoporus there are cellular processes enveloping reserve material. Presumably, these observations indicate a so-called "filtration of nutritive yolk" (Korschelt \& Heider, 1909) that might represent an additional mode for the transfer of yolk-containing cellular fragments from blastocoel into gastrocoel.
\end{abstract}

\section{EINLEITUNG}

Die Aktinie Anemonia sulcata entwickelt dotterreiche, bestachelte Oocyten, die größere Mengen an symbiontischen Zooxanthellen enthalten (Schmidt \& Schäfer, 1980; Schäfer, 1983b; Schäfer, 1984). Sie ist getrenntgeschlechtlich und ovipar (Graeffe, 1884; Stevenson, 1935; Pax, 1936); ihre Oocyten lassen nach der Besamung drastische Veränderungen in der Rindenregion erkennen, die auf eine heftige Corticalreaktion zurückzuführen sind. In der Folge entsteht eine den jungen Keim umhüllende Schicht, die sich aus Teilen des peripheren Plasmas, extruierten Zooxanthellen, der feinverteilten Matrix ehemaliger Corticalgranula sowie abgesprengten Stacheln zusammensetzt (Schäfer, 1984).

Die Ontogenese der Actiniaria wurde bisher nur lichtmikroskopisch untersucht (Lacaze Duthiers, 1872; Appellöf, 1900; Cary, 1910; Gemmill, 1920; Nyholm, 1943; Chia \& Spaulding, 1972; Spaulding, 1974; Siebert, 1974). Bei Embryonen, die aus dotterrei- 
chen Oocyten hervorgehen, kann nicht selten eine Verzögerung der Blastomerensonderung beobachtet werden (Siewing, 1969), wobei im Laufe der weiteren Entwicklung ein lockerer Verband dotterhaltiger Zellen im Blastocoel (Gemmill, 1922: "trophenchyme") entsteht. Im Zuge der Entodermablösung erfährt die klassische Embolie hierdurch Abwandlungen bis zur Unkenntlichkeit, was in der Bildung stark modifizierter Invaginationsgastrulae endet (vgl. Cary, 1910).

Abgesehen von einigen Arbeiten, die sich u. a. mit speziellen Fragestellungen wie beispielsweise der Corticalreaktion befassen (Dewel \& Clark, 1974; Schäfer \& Schmidt, 1980; Schäfer, 1984; Larkman \& Carter, 1984), sind noch keine elektronenoptischen Befunde über die Ontogenese der Actiniaria publiziert. Daher soll im Rahmen der vorliegenden Arbeit die Ultrastruktur der Embryonen von Anemonia sulcata näher untersucht werden, wobei den Blastulae und Gastrulae besondere Aufmerksamkeit gewidmet wird.

\section{MATERIAL UND METHODEN}

Die untersuchten Embryonen stammten von 20 unmittelbar vor der Fortpflanzungsperiode gesammelten Tieren, die in Seewasseraquarien des I. Zoologischen Instituts der Universität Erlangen ablaichten. Ein Teil der Anemonia-Keime wurde fixiert, der Rest wurde für Lebendbeobachtungen in kleinen Aquarien (2 Liter) weitergezüchtet. Die für die elektronenoptischen Untersuchungen angewandten Methoden können Teil I der Arbeit (Schäfer, 1984) entnommen werden. Die lichtmikroskopischen Befunde wurden anhand von Lebendmaterial und Semidünnschnitten erhoben.

\section{ERGEBNISSE}

\section{Frühe Stadien der Ontogenese}

Nach der Besamung bleiben die erwarteten Furchungsteilungen aus. Binnen der folgenden 24 Stunden fallen lichtmikroskopisch lediglich Veränderungen in der peripheren Region des Keimes auf, wobei die ursprüngliche, regelmäßige Anordnung der Stacheln als Folge der an anderer Stelle bereits näher beschriebenen Corticalreaktion verlorengeht (Schäfer, 1984). Bei etwa zwei Drittel der ca. 36 Stunden alten Keime konnte beobachtet werden, daß sie ihre nahezu kugelige Form nicht beibehalten, sondern eine Streckung sowie oftmals auch eine leichte Abplattung erfahren. In der Folge kommt es zur Bildung recht irregulär aufgebauter Embryonen (Abb. 1, 2). Knapp die Hälfte dieser Keime ging zugrunde. Die übrigen nehmen jedoch im Laufe der folgenden 6-10 Stunden wieder die ursprüngliche, mehr oder weniger kugelige Gestalt an. Diese Embryonen entwickelten sich ebenso wie diejenigen, bei denen keine derartigen Formveränderungen zu verzeichnen waren, weiter und können dann von letzteren nicht mehr unterschieden werden.

Rasterelektronenmikroskopisch sind an der Oberfläche der Keime lange Mikrovilli nachzuweisen. Gruppenweise aneinandergelagert werden Koni gebildet (Abb. 3), die sich aus 15-30 solcher Mikrovilli aufbauen und lichtmikroskopisch den Stacheln frisch abgelaichter Oocyten ähneln. Diese sind jedoch viel regelmäßiger angeordnet und aufgebaut (vgl. Schmidt \& Schäfer, 1980; Schäfer, 1984).

Spätestens nach 48 Stunden ist die allmähliche Bildung unregelmäßig verlaufender 
Einkerbungen an der Oberfläche des Embryos zu verzeichnen (Abb. 5). Ich fand keine Anhaltspunkte dafür, daß zwischen letzteren und Blastomerengrenzen irgendeine Beziehung besteht: die Entwicklung des Anemonia-Keimes ist von einer Verzögerung der Blastomerensonderung begleitet, was erklärt, warum lichtmikroskopisch keine Furchung beobachtet werden konnte. Das Cytoplasma der 2 Tage alten Keime ist mit Dottergranula erfüllt, peripher im Bereich der Einkerbungen liegen noch Corticalgranula (Abb. 8), die ihr Internum nach der Besamung nicht durch Exocytose in das umgebende Medium entleert haben. In einem von Speicherstoffen weitgehend freien Bereich finden sich Fragmente vielfach gewundener Doppelmembranen, die Porenkomplexe aufweisen (Abb. 6, 7).

\section{Blastula}

Im Laufe der Frühentwicklung haben asynchron und in unregelmäßigen Abständen Kernteilungen stattgefunden. Der größte Teil der Nuclei drängt in die äußeren Regionen des Keimes, Mit dem Auftreten von Zellgrenzen wird das Stadium der Blastula erreicht. Die frühen Blastulae stellen einen Verband aus Zellen und dotterhaltigen, kernlosen Zellfragmenten dar (Abb.9). In dieser Phase werden in den Blastomeren vom GolgiApparat elektronendichte Pigmentgranula abgeschieden (Abb. 10), die sich jeweils peripher anreichern (Abb. 11).

Allmählich erfolgt unter diesen Voraussetzungen die epitheliale Anordinung des Blastoderms, mit der eine Auflösung des engen Verbandes der dotterhaltigen Zellfragmente einhergeht (Abb. 12). Letztere werden auch vom Blastoderm ins Innere des Keimes abgespalten, so daß die Blastodermzellen, wenn überhaupt, nur einige wenige Dottergranula enthalten. Im übrigen kennzeichnen einzelne annulierte Lamellen, Vakuolen, Lipidtröpfchen sowie vor allem die peripher angereicherten Pigmentgranula die innere Struktur der Blastodermzellen. An der Oberfläche sind die oben beschriebenen, langen Mikrovilli vorhanden; Flagellen wurden dagegen noch nicht entwickelt. Auch Zellgrenzen sind äußerlich vorerst nicht erkennbar. Furchen, die im Gegensatz zu den schon früher beobachteten Einkerbungen (vgl. Abb. 5) auch tatsächlich Blastomerengrenzen repräsentieren (Abb. 13), treten erst im Laufe des 3. Tages nach der Besamung auf.

Ein typisches Blastocoel existiert nicht (Abb. 12-15). Dieser Raum wird von einem lockeren Verband aus Zellen bzw. Zellfragmenten eingenommen, wobei im wesentlichen drei Typen unterschieden werden können (Abb. 14). Größtenteils handelt es sich hierbei um die bereits mehrfach angesprochenen dotterhaltigen Zellfragmente, die, wie anhand von Serienschnitten nachgewiesen werden konnte, keine Zellkerne, auch keine degenerativen, enthalten. Sie sind mit heterogenen Speicherstoffgranula erfüllt, die sich aus einem osmiophilen Anteil sowie Kugeln mit lipidähnlichem Kontrast zusammensetzen ("Komplexdotter B"; vgl. Schäfer, 1983a). Daneben treten Zellen mit Kern und wenig Dotter auf. Schließlich sind noch solche Zellfragmente zu beobachten, die eine Zooxanthelle bergen (Abb. 14). Diese, das Innere des Keimes ausfüllenden Elemente sind stellenweise dicht gedrängt gelagert (Abb. 13). Andererseits bleiben lokal auch miteinander kommunizierende Spalträume erhalten (Abb. 12), deren Gesamtheit mit dem Blastocoel zu homologisieren wäre. In diesen Blastocoelspalten finden sich auch einzelne Dottergranula, Lipidtröpfchen, Schleim, Zooxanthellen und Membranfrag- 

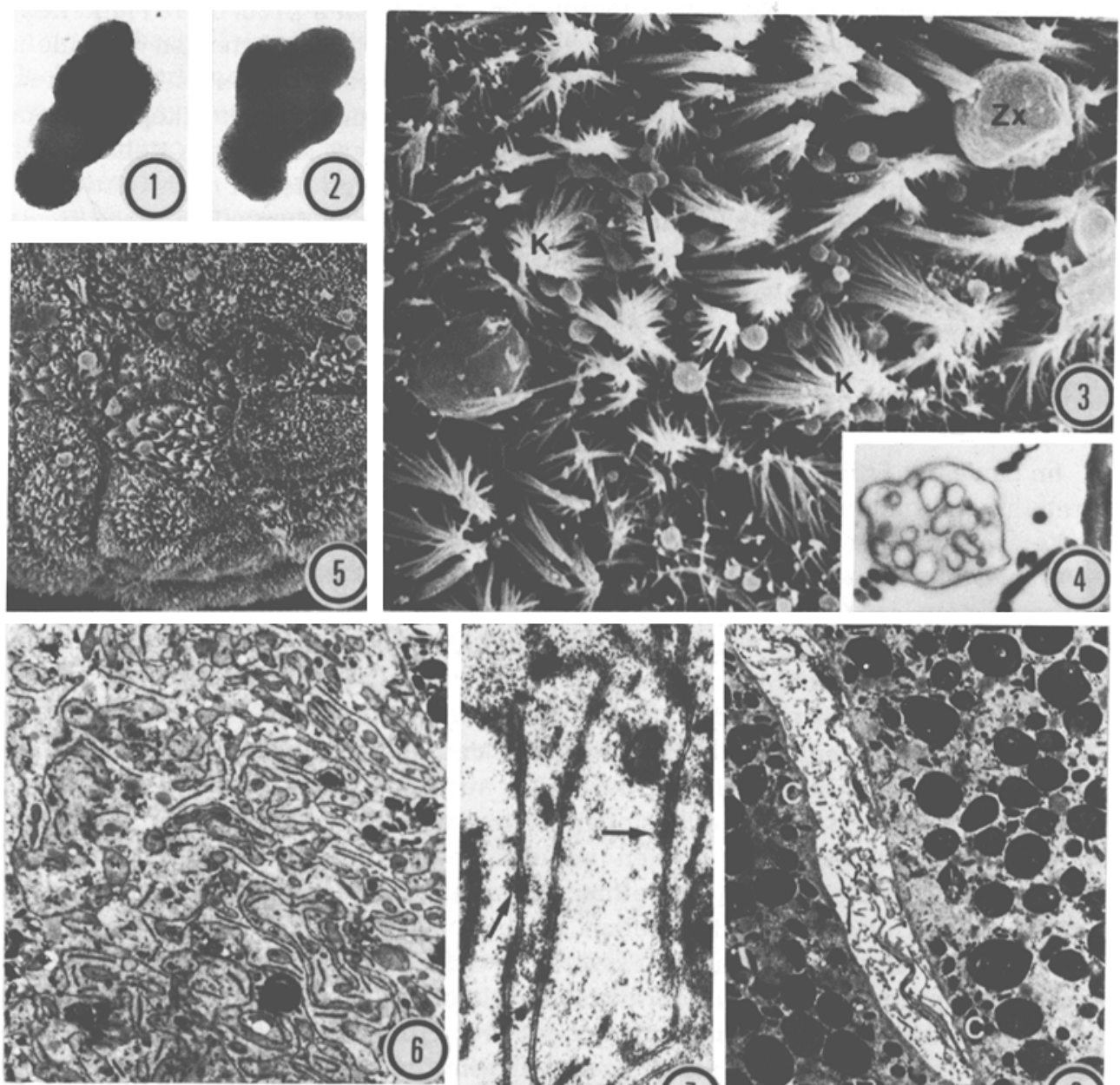
s.

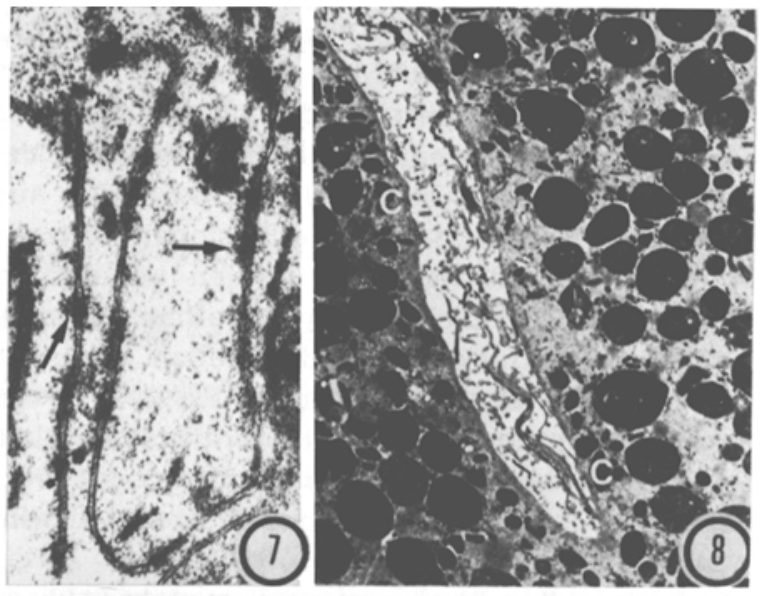

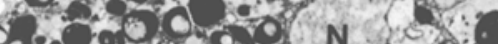

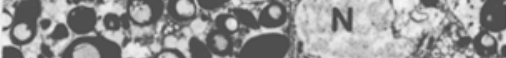
- 0 on 40 to

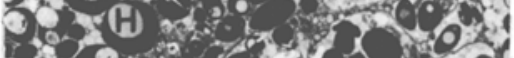

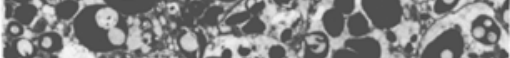

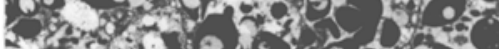

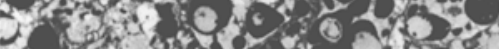
ryos bo 0 ons

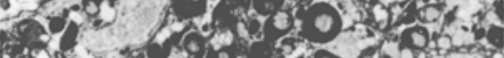

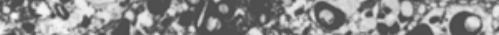

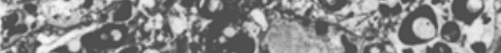

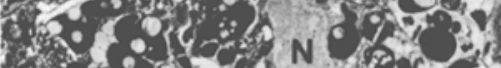

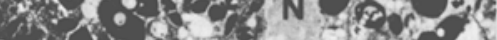

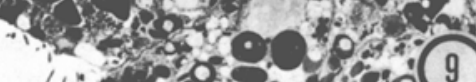

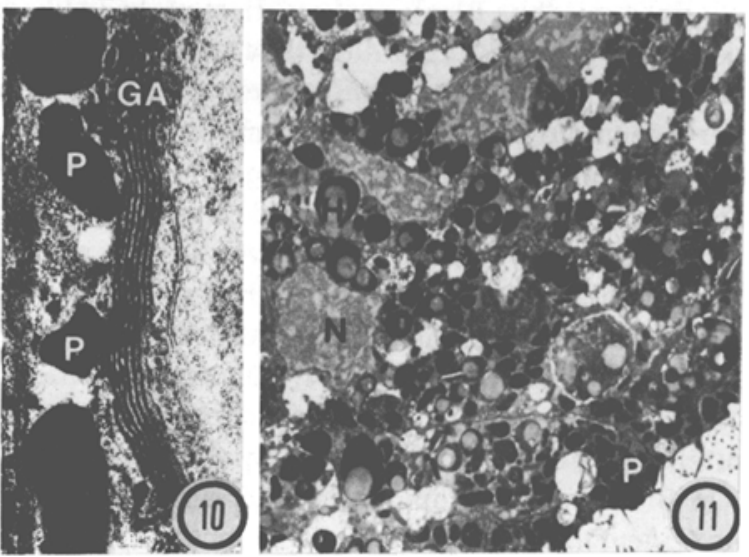


mente, wobei es sich jeweils um Zerfallsprodukte der oben genannten Zellen bzw. Zellteile handeln dürfte.

In späten Blastulae treten intranucleäre annulierte Lamellen in Erscheinung. In der Regel werden Einzellamellen gebildet, worin ein Unterschied zu den cytoplasmatischen annulierten Lamellen zu sehen ist, die angehäuft als mehr oder weniger große Stapel vorkommen. Die bisweilen noch erkennbare Kontinuität zwischen Kernmembran und intranucleären Lamellen (Abb. 16) läßt vermuten, daß letztere durch Abspaltung von der Kernhülle entstehen. Danach liegen sie isoliert im Chromatin, wobei ihre ursprüngliche, durch Doppelmembran und Porenkomplexe charakterisierte Struktur noch lange Zeit erhalten bleibt (Abb. 17). Mit der Blastula ist beim Anemonia-Keim nunmehr das Stadium erreicht, ab dem auf zellulärer Ebene keine weitere Differenzierung bis zum Abschluß der Gastrulation zu verzeichnen ist.

\section{Gastrula}

Nach dem Kollabieren des Germinalvesikels, durch dessen exzentrische Lage der animale Pol gekennzeichnet war, konnte während der gesamten Entwicklung bis zur Blastula optisch keine Polarität des Keimes nachgewiesen werden. Mit der Gastrulation tritt jedoch die monaxon-heteropolare Körperachse in Erscheinung, die von diesem Zeitpunkt an beibehalten wird.

Nach ca. 3 Tagen weisen die annähernd kugeligen Embryonen eine deutliche Eindellung auf (Abb. 18), deren Durchmesser anfangs bis zu $180 \mu \mathrm{m}$ betragen kann. Bisweilen plattet sich der Kern auffällig ab und ist am ehesten mit einer rundlichen Schale zu vergleichen, die eine konvexe und, gegenüberliegend, eine konkave Seite aufweist. Diese Vorgänge signalisieren den Beginn der Gastrulation. Die Zellen der

Abb. 1, 2. Anemonia. 36 Stunden alte, irregulär aufgebaute Embryonen; $45: 1$

Abb. 3. Oberfläche eines 36 Stunden alten Anemonia-Keimes mit extruierten Zooxanthellen (Zx) und multivakuolären Körperchen (Pfeile); lange Mikrovilli lagern sich zu auffälligen Koni (K) zusammen; $2050: 1$

Abb. 4. Multivakuoläres Körperchen; $11600: 1$

Abb. 5. Oberfläche eines 36 Stunden alten Anemonia-Keimes mit unregelmäßig verlaufenden Einkerbungen; $310: 1$

Abb. 6. Membranfragmente; $2900: 1$

Abb. 7. Wie Abb. 6, Ausschnitt; Pfeile deuten auf Porenkomplexe; 24 300:1

Abb. 8. Einkerbung an der Oberfläche des Keimes. C: Corticalgranula; $2500: 1$

Abb. 9. Periphere Region einer frühen Blastula. H: heterogene Dottergranula, N: Nucleus; $2300: 1$

Abb. 10. Sekretion von Pigmentgranula (P) durch den Golgi-Apparat (GA); $20500: 1$

Abb. 11. Periphere Region einer Blastula. H: heterogene Dottergranula, N: Nucleus, P: Pigmentgranula; $2200: 1$ 
konvexen Außenfläche werden zu Ektoderm, die der konkaven Innenfläche bzw. die im Bereich der oben beschriebenen Eindellung zu Entoderm. Die in diesem Stadium noch weite Öffnung des Urdarmes nach außen, der Blastoporus, verengt sich im Laufe der folgenden 12 Stunden stetig (Abb. 19), womit eine pfropfartige Einsenkung des Ento-
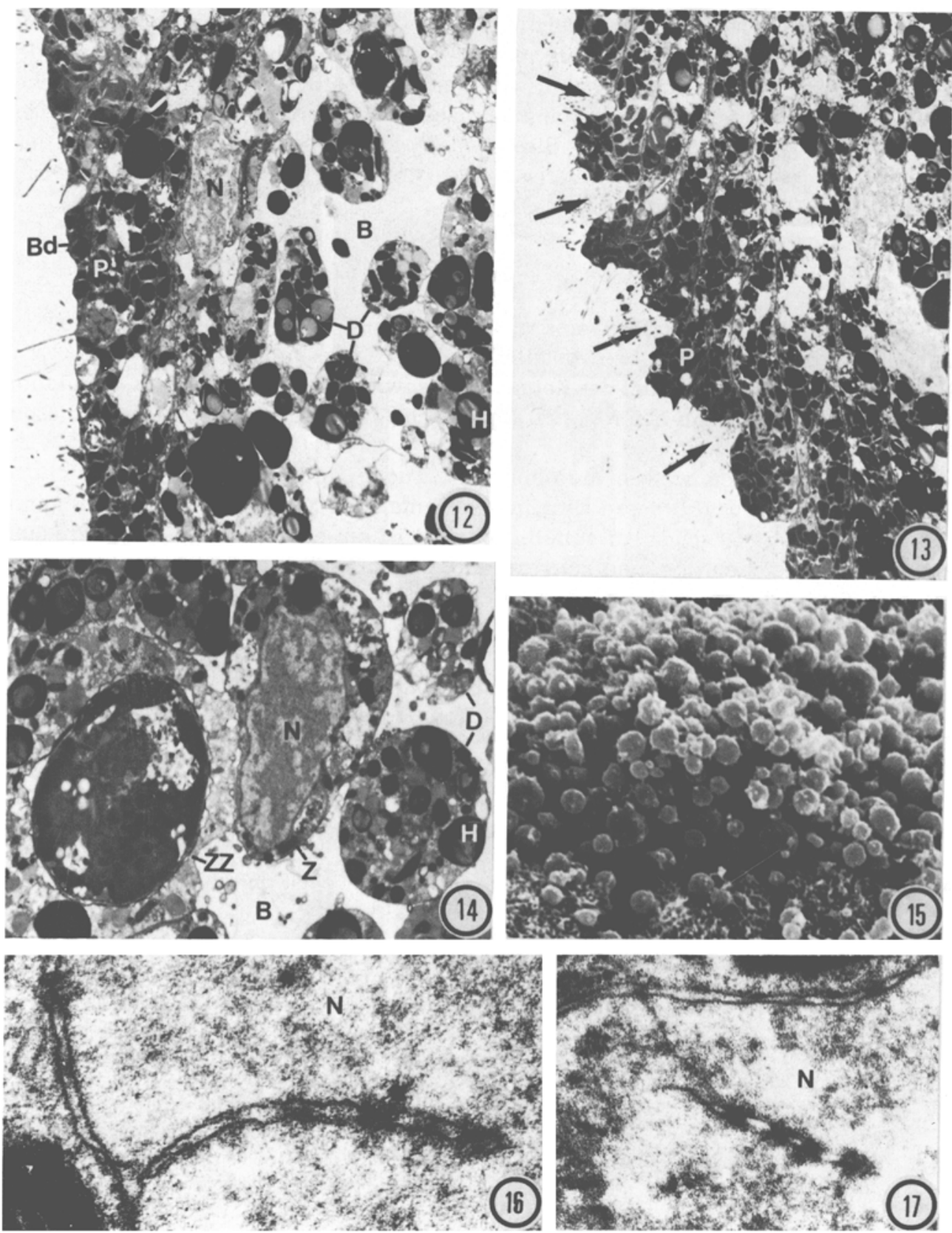
derms einhergeht. Hierbei nähert sich das Urdarmdach der Innenseite des apikalen Ektoderms bis auf ca. $80 \mu \mathrm{m}$. Gleichzeitig nehmen die Keime wieder ihre mehr oder weniger kugelige Form an. Der Durchmesser des Blastoporus hat sich mittlerweile auf $20-40 \mu \mathrm{m}$ verkleinert. Nach Ablauf dieses Prozesses ist die eigentliche Invagination des Entoderms abgeschlossen. Die Grundgestalt der becherförmigen Gastrula ist verwirklicht. Jedoch umfaßt der Urdarm zunächst nur einen Bruchteil des Volumens der primären Leibeshöhle, weil das invaginierende Entoderm das vorwiegend mit Speicherstoffen erfüllte Blastocoel zunächst nicht verdrängen kann.

Ca. 12 Stunden nach Beginn der Gastrulation finden sich im Urdarm erstmals dotterhaltige Zellteile. Dies zeigt an, daß eine neue Phase begonnen hat: die Annäherung der beiden Keimblätter, wobei sich das Gastrocoel in dem Maße vergrößert, wie das Blastocoel schrumpft. Dies wiederum setzt voraus, daß die Zellen bzw. die Zellfragmente, die das Blastocoel erfüllen, allmählich ins Gastrocoel übertreten. In diesem fortgeschrittenen Stadium sind die Anemonia-Gastrulae folgendermaßen charakterisiert; die monaxon-heteropolare Körperachse verläuft durch den Blastoporus und den apikalen Pol, dessen Zellen keine besondere Differenzierung erkennen lassen (Abb. 22). Dem Blastoporus schließt sich ein Kanal an, der mittlerweile nur noch einen Durchmesser von $10-20 \mu \mathrm{m}$ aufweist und sich trichterartig in das zentrale Gastrocoel öffnet (Abb. 23, 24). Letzteres wiederum bildet mehrere Aussackungen, meist sind 3 bis 5 radiäre Divertikel vorhanden. Darüber hinaus kann, oft etwas außerhalb der Körperachse liegend, ein apikales Gastrocoeldivertikel beobachtet werden (Abb. 24).

Im Vergleich zum Ektoderm bilden die außerordentlich polymorphen Zellen des Entoderms einen relativ lockeren Verband, so daß Blastocoelspalten und Gastrocoel an vielen Stellen nur durch dünne Plasmafortsätze voneinander getrennt sind (Abb. 20, 24-26). In der Beschaffenheit des Cytoplasmas gleichen die Zellen des Ekto- und Entoderms immer noch denen des Blastoderms (vgl. Abb. 12 mit Abb. 20, 22, 25-28). Die Ektodermzellen haben nach wie vor vereinzelt Mikrovilli, die sich konisch zu stachelähnlichen Gebilden aneinanderlagern (Abb. 22). Dies trifft für das Entoderm allerdings nicht mehr zu. Der Mikrovillisaum als solcher bleibt jedoch zum Gastrocoel hin erhalten

Abb. 12. Periphere Region einer Blastula. B: Blastocoelspalten, Bd: Blastoderm, D: dotterhaltige Zellfragmente, H: heterogene Speicherstoffgranula, N: Nucleus, P: Pigmentgranula; $2880: 1$

Abb. 13. Periphere Region einer späten Blastula; Blastomerengrenzen erkennbar (Pfeile). P: Pigmentgranula; 2570:1

Abb. 14. Die häufigsten Typen von Zellen bzw. Zellfragmenten, die das Blastocoel ausfüllen. B: Blastocoelspalten, D: dotterhaltige Zellfragmente, $H$ : heterogene Speicherstoffgranula, $N$ : Nucleus, $\mathrm{Z}$ : Zelle, ZZ: Zellfragment mit Zooxanthelle; $7100: 1$

Abb. 15. Einblick ins Blastocoel nach teilweisem Entfernen des Blastoderms; das Blastocoel ist von einem mehr oder weniger lockeren Verband annähernd kugeliger Elemente (vgl. Abb. 12, 14, 20) erfült; $970: 1$

Abb. 16. Bildung einer intranucleären, annulierten Lamelle durch Abspaltung von der Kernmembran. N: Nucleoplasma; 50 100:1

Abb. 17. Intranucleäre, annulierte Lamelle. N: Nucleoplasma; $37000: 1$ 

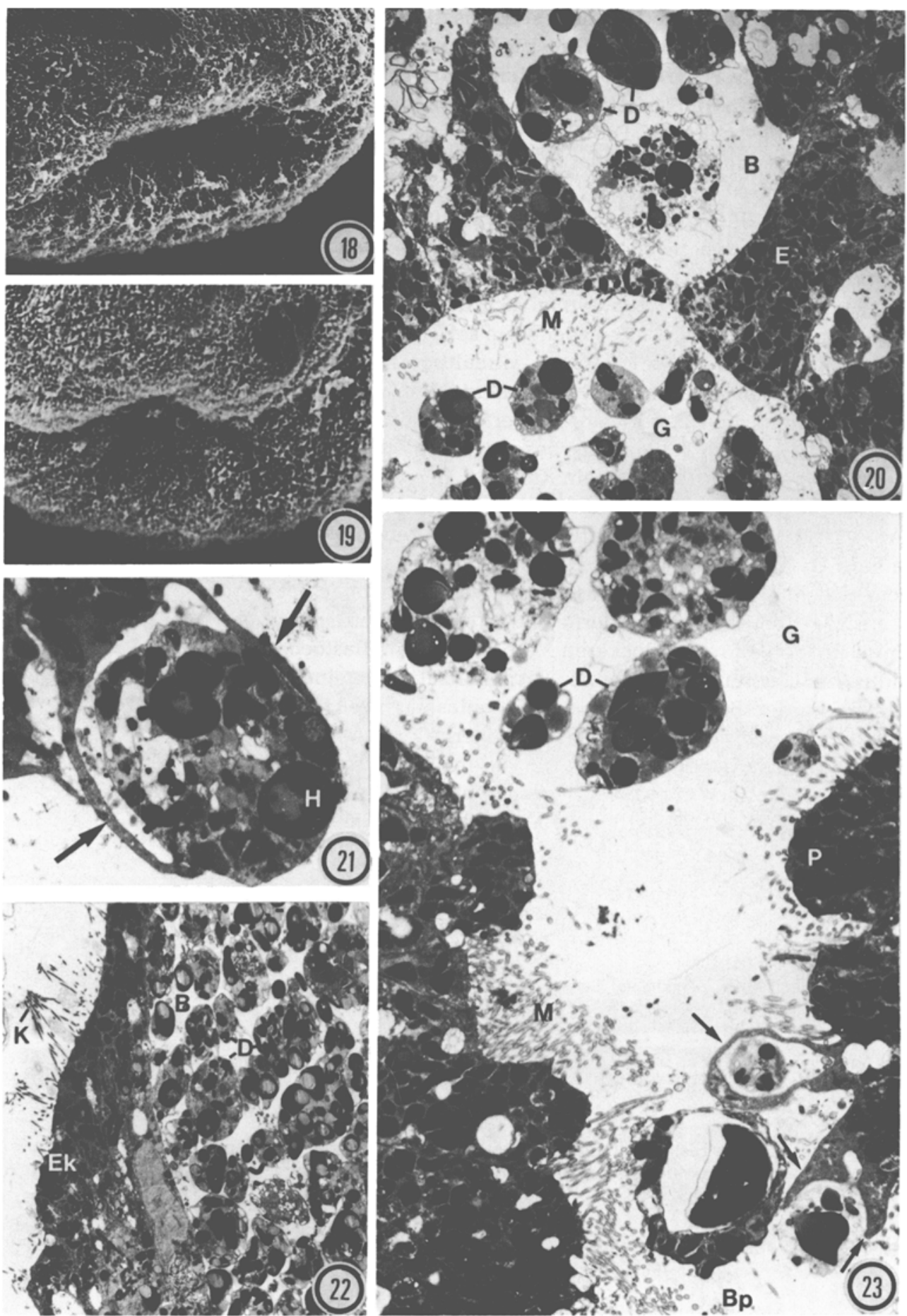
(Abb. 25, 26) und erscheint in der Region des Blastoporus besonders ausgeprägt (Abb. 23, 28). Dagegen läßt sich als allgemeine Tendenz beobachten, daß die Zellen beider Keimblätter auf der zum Blastocoel hingewandten Seite nur wenig, meist gar keine Mikrovilli entwickeln (Abb. 20, 22).

Inzwischen sind nahezu an der gesamten Oberfläche der Gastrulae Furchen in Erscheinung getreten, die auch tatsächlich Zellgrenzen repräsentieren. Dies gilt auch für die Blastoporusregion (Abb. 23, 28), deren Zellen außer dem dichten Mikrovillisaum noch eine weitere Besonderheit aufweisen. Sie bilden Ausläufer, die im Anschnitt wie makrovilliartige Fortsätze wirken und Dotterkugeln oder dotterhaltige Zellfragmente einhüllen bzw. zangenartig umgreifen (Abb. 21, 23). Es läßt sich gegenwärtig nur schwer beurteilen, ob die Zellen der Blastoporusregion die Nährsubstanzen phagocytieren oder ob umgekehrt ein Prozeß im Gange ist, bei dem dotterhaltige Zellteile ins Gastrocoel entlassen werden, was in diesem Fall als möglicher Mechanismus für den Transport von Speicherstoffen aus dem Blastocoel ins Gastrocoel in Betracht zu ziehen wäre. Letzteres wird vor allem in den Gastrocoeldivertikeln allerdings auf viel einfachere Art ermöglicht. An einigen Stellen sind im ohnehin lockeren Verband der Entodermzellen Lücken nachzuweisen, über die Blastocoelspalten und Gastrocoel direkt miteinander in Verbindung stehen, wodurch vor allem größere, dotterhaltige Zellteile ins Gastrocoel gelangen (Abb. 27).

In der Endphase der Gastrulation geht mit dem oben geschilderten Vorgang eine leichte Streckung des Keimes einher, der langsam eine ovale bis birnenförmige Gestalt annimmt. Nach den bisherigen Befunden ist eine weitere Differenzierung auf zellulärer Ebene und Mesogloeabildung erst dann zu verzeichnen, wenn nahezu alle dotterhaltigen Zellfragmente vom Blastocoel ins Gastrocoel übergetreten sind.

\section{Zooxanthellen}

Der Zerfall des Deutoplasmas in dotterhaltige Zellfragmente und die dadurch bedingten mechanischen Einwirkungen auf den Periplasten und das die Zooxanthellen umgebende Plasma bewirkt, daß letztere aus ihrem Verband mit anderen Zellen isoliert

Abb. 18. Blastoporus ca. 2 Stunden nach Gastrulationsbeginn; $380: 1$

Abb. 19. Blastoporus ca. 12 Stunden nach Gastrulationsbeginn; $380: 1$

Abb. 20. Grenzregion Blastocoel/Gastrocoel (vgl. Abb. 24). B: Blastocoelspalten, D; dotterhaltige

Zellfragmente, E: Entodermzelle mit Pigmentgranula, G: Gastrocoel, M: Mikrovilli; $2170: 1$

Abb. 21. Dotterhaltiges Zellfragment umklammert von makrovilliartigen Zellfortsätzen (Pfeile). H: heterogene Dottergranula; $6130: 1$

Abb. 22. Periphere Region einer Gastrula nahe des animalen Pols (vgl. Abb. 24). B: Blastocoelspalten, D: dotterhaltige Zellfragmente, Ek: Ektodermzelle mit Pigmentgranula, K: Konus aus zusammengelagerten Mikrovilli; 2260:1

Abb. 23. Blastoporusregion (vgl. Abb. 24); Pfeile deuten auf makrovilliartige Zellfortsätze. Bp: Blastoporus, D: dotterhaltige Zellfragmente, G: Gastrocoel, M: Mikrovilli, P: Pigmentgranula; 3420 : 1 


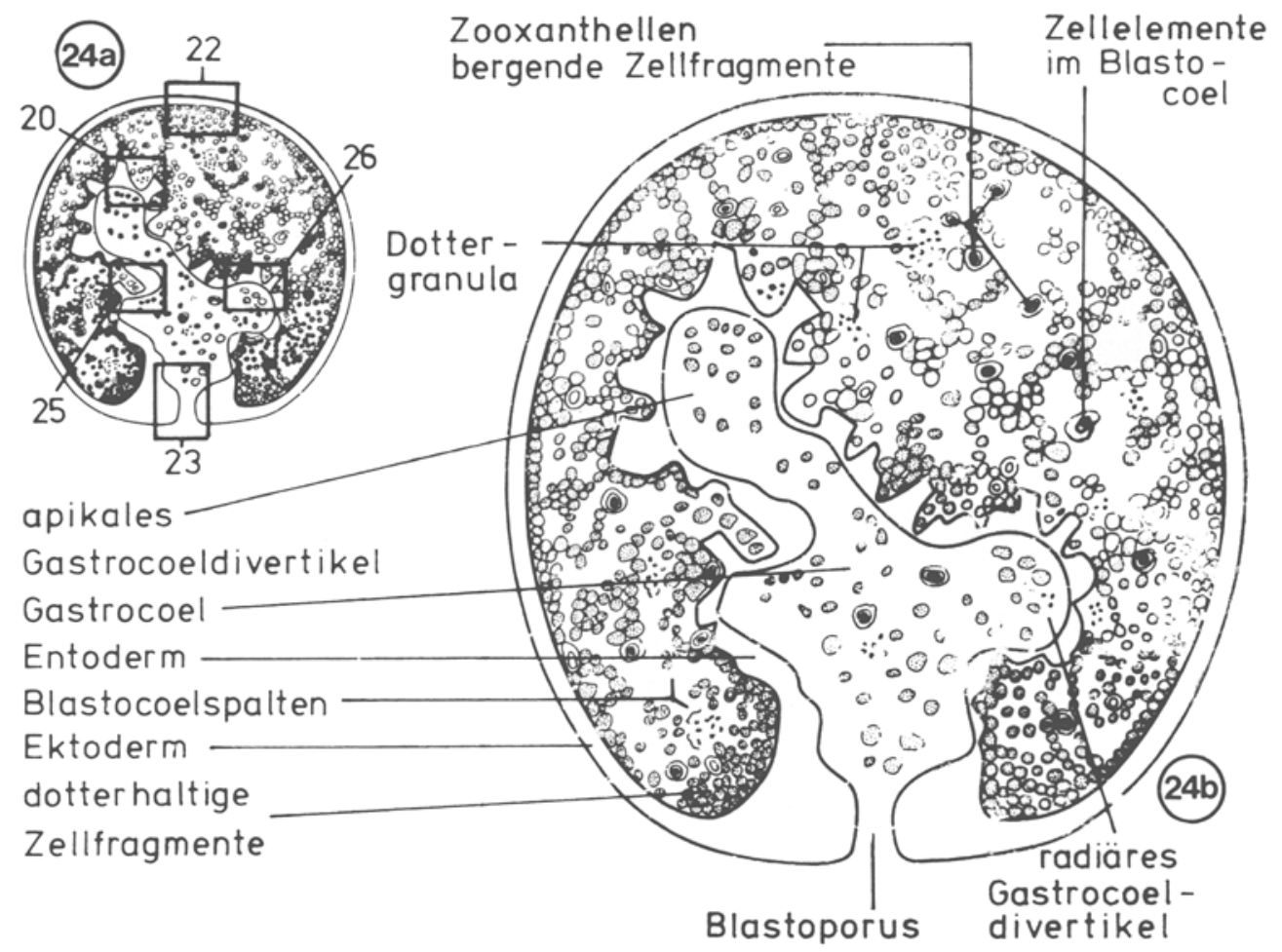

Abb. 24. Gastrula von Anemonia sulcata. (a) Ubersicht: Zahlen und Markierungen verweisen auf elektronenoptische Abbildungen. (b) Gastrula längs, gezeichnet nach einem Semidünnschnitt; $280: 1$

werden. Viele gelangen über Interzellularen zur Peripherie des Keimes, werden ausgestoßen und treten schließlich an der Oberfläche der Embryonen in Erscheinung (Abb. 3). Ferner sammelt sich dort eine größere Anzahl kleiner, annähernd kugeliger Gebilde an. Es handelt sich hierbei um multivakuoläre Körperchen (Abb. 3, 4), die durch Exocytose nach außen gelangt sind und sich vorübergehend zwischen den Mikrovilli verfangen.

Einige Zooxanthellen verbleiben jedoch in voluminösen, relativ dotterarmen Zellteilen (Abb. 14), die vorübergehend im Blastocoel, manchmal auch im Gastrocoel (Abb. 28), anzutreffen sind. Sie werden größtenteils in die Epithelien reintegriert. Dies gewährleistet letztendlich, daß die Zooxanthellen des Muttertieres an die Individuen der nächsten Generation, in denen sie sich wieder schnell vermehren und ausbreiten, weitergegeben werden.

\section{DISKUSSION}

\section{Die Frühentwicklung bis zur Blastula}

Die frühen Entwicklungsstadien des Anemonia-Keimes sind durch folgende Charakteristika gekennzeichnet: (1) eine heftige Corticalreaktion nach der Besamung (vgl. 


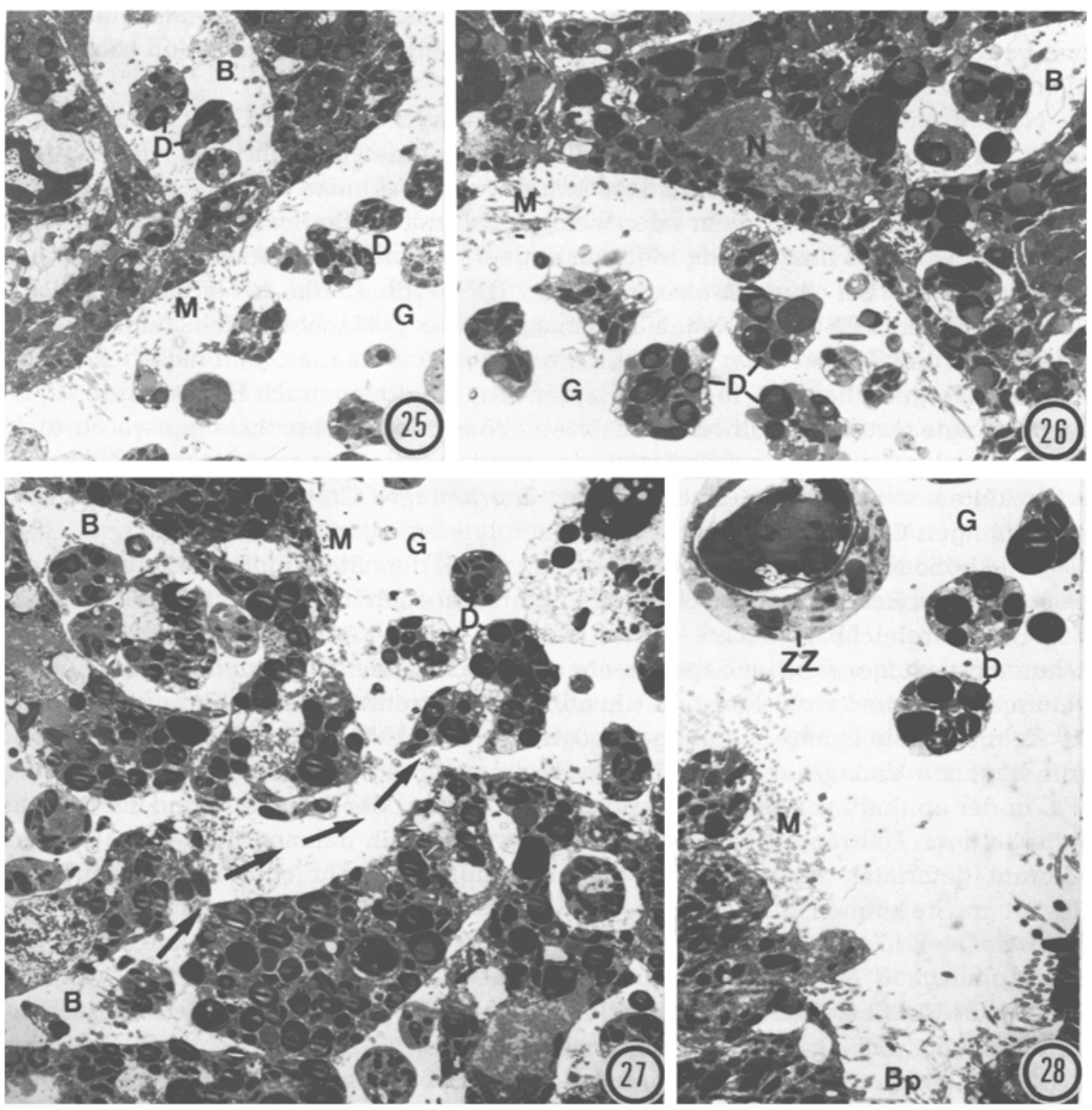

Abb. 25. Grenzregion Blastocoel/Gastrocoel (vgl. Abb. 24). B: Blastocoelspalte, D; dotterhaltige Zellfragmente, G: Gastrocoel, M: Mikrovilli; $1860: 1$

Abb. 26. Grenzregion Blastocoel/radiäres Gastrocoeldivertikel (vgl. Abb. 24). B: Blastocoelspalte, D: dotterhaltige Zellfragmente, G: Gastrocoel, M: Mikrovilli, N: Kern einer Entodermzelle; $2430: 1$

Abb, 27. Blastocoelspalten und Gastrocoel in Verbindung; Pfeile kennzeichnen den vermuteten Weg, über den dotterhaltige Zellfragmente von den Blastocoelspalten ins Gastrocoel übertreten. B: Blastocoelspalten, D: dotterhaltige Zellfragmente, G; Gastrocoel, M: Mikrovilli; $2210: 1$

Abb. 28. Blastoporusregion einer älteren Gastrula. Bp: Blastoporus, D: dotterhaltige Zellfragmente, G: Gastrocoel, M: Mikrovilli, ZZ: Zellfragment mit Zooxanthelle; 2600:1 
Schäfer, 1984), (2) die vorübergehend irreguläre äußere Gestalt eines Teiles der Embryonen (Abb. 1, 2), (3) die Anhäufung atypischer annulierter Lamellen (Abb. 6, 7), (4) eine Verzögerung der Blastomerensonderung.

Neuere elektronenmikroskopische Untersuchungen über die Corticalreaktion bei Actiniaria-Oocyten haben zu unterschiedlichen Ergebnissen geführt. Bei Alfredus lucifugus (Schäfer \& Schmidt, 1980) und Actinia fragacea (Larkman \& Carter, 1984) läßt sich lediglich beobachten, wie mehr oder weniger zahlreiche Corticalgranula ihren Inhalt durch Exocytose ins umgebende Medium entleeren. Eine sehr heftige Corticalreaktion wurde dagegen bei Bunodosoma cavernata (Dewel \& Clark, 1974) und Anemonia sulcata (Schäfer, 1984) beschrieben. Larkman \& Carter (1984) bezweifeln aufgrund ihrer eigenen Befunde an Actinia, daß es sich bei Bunodosoma um einen natürlichen Entwicklungsvorgang handelt. In der Tat lassen sich mögliche, durch Hälterung in Aquarien bedingte Artefakte schwer abschätzen. Wie jedoch lichtmikroskopisch an nicht fixierten und isoliert weitergezüchteten Anemonia-Embryonen verfolgt werden konnte, entwickelten sich solche Keime, die eine ausgeprägte Corticalreaktion zeigten, zu lebensfähigen Gastrulae und Larven, die zu Polypen metamorphosierten.

Eine hohe Mortalität (ca. $30 \%$ ) war dagegen in dem Stadium festzustellen, wo ein großer Teil der Embryonen den oben näher beschriebenen irregulären Aufbau erkennen läßt. Eine vergleichbare äußere Gestalt haben Sagartia-Keime, die Sembrat (1956) im Rahmen morphogenetischer Experimente erhalten hat. Die bei Anemonia beobachteten Deformationen sind wohl ebenfalls unnatürlich. Sie treten zu einem Zeitpunkt auf, wo die Keime auf die Laborbedingungen möglicherweise besonders sensibel reagieren, da grundlegende Umlagerungen in diesem Stadium zu verzeichnen sind, die schließlich u. a. in der epithelialen Anordnung des Blastoderms an der Peripherie und der Bildung dotterhaltiger Zellfragmente im Innern enden. Immerhin nehmen über die Hälfte der aberrant geformten Keime wieder die ursprüngliche, mehr oder weniger kugelige Gestalt an. Sie können im Laufe der weiteren Entwicklung nicht mehr von den normalen Embryonen, d. h. von solchen, die keine auffälligen Deformationen zeigten, unterschieden werden.

Annulierte Lamellen (Porenlamellen) sind in Oocyten, embryonalen und schnell wachsenden Zellen häufig anzutreffen (Wischnitzer, 1970). Meist bilden sie Stapel parallel übereinander lagernder Lamellen, wie sie mittlerweile auch in Anthozoenoocyten nachgewiesen sind. Sie spielen bei der Bildung des rauhen Endoplasmatischen Reticulums eine Rolle (Schäfer, 1983a). Die in Anemonia-Keimen beobachtete unregelmäßige Anordnung der vielfach gewundenen Porenlamellen (Abb. 6) ist jedoch ungewöhnlich. Eine Beziehung zu anderen Zellorganellen war nicht festzustellen. Somit liegt die Vermutung nahe, daß diese atypischen annulierten Lamellen, möglicherweise Fragmente der Kernhülle, keine Funktion mehr ausüben.

Der junge Anemonia-Keim läßt eine Verzögerung der Blastomerensonderung erkennen, wie sie bei solchen Cnidaria, deren Oocyten einen gewissen Dotterreichtum aufweisen, vorkommt (Siewing, 1969; Mergner, 1971). Oft treten Blastomerengrenzen im Stadium 16, bei Actiniaria wie Adamsia palliata und Sagartia troglodytes bereits im Stadium 4 in Erscheinung (Gemmill, 1920; Nyholm, 1943). Vergleichbar regelmäßige Entwicklungsvorgänge und eine so relativ frühe Blastomerensonderung konnte bei Anemonia nicht beobachtet werden. Dies ist hauptsächlich auf die asynchron ablaufenden Teilungen der Kerne zurückzuführen. Letztere drängen nur langsam zur Peripherie 
des Keimes, entsprechend werden erst bei 2-3 Tage alten Embryonen auch an deren Oberfläche Zellgrenzen sichtbar (Abb. 13).

\section{Blastula}

Bei Anemonia führt die Ontogenese nicht zur typischen Coeloblastula. Es kommt vielmehr zur Bildung dotterhaltiger Zellfragmente, die nach innen abgesondert werden und weite Teile des Blastocoels ausfüllen. Dieses "Trophenchym", wie Gemmill (1922) den in erster Linie aus dotterhaltigen Zellfragmenten bestehenden Verband bezeichnet, ist bei Anemonia besonders ausgeprägt (vgl. Appellöf, 1900; Cary, 1910; Nyholm, 1943). Dementsprechend finden sich im Inneren der Blastula nur einige Spalträume (Abb. 12-15) als Reste des Blastocoels.

Mit der Blastula erreicht der Anemonia-Keim das Stadium, ab dem zunächst keine weitere Differenzierung auf zellulärer Ebene zu beobachten ist. Insbesondere werden noch keine Cilien gebildet. Diese treten bei späten Gastrulae in Erscheinung, worauf die Embryonen erste Rotationsbewegungen ausführen (Schäfer, in Vorbereitung). Ähnliches trifft offensichtlich für Cribrinopsis fernaldi zu (Siebert \& Spaulding, 1976), während bei anderen Actinaria bewegliche Blastulae beschrieben werden (Gemmill, 1920, 1922; Spaulding, 1974).

Auffallend häufig liegen im Karyoplasma der Blastodermzellen einzelne intranucleäre annulierte Lamellen (Abb. 17). Uber ihre Genese und Bedeutung ist nur wenig bekannt (vgl. Wischnitzer, 1970). Die Befunde an Anemonia-Embryonen lassen vermuten, daß die Porenlamellen unmittelbar von der Kernhülle abgetrennt werden (Abb. 16), eine Funktion kann ihnen jedoch nicht zugeschrieben werden.

\section{Gastrula}

Das Innere der Blastulae ist bei Anemonia sulcata weitgehend mit Speicherstoffen erfüllt, wodurch das Blastocoel bis auf einige Spalträume reduziert ist. Somit erscheinen die Voraussetzungen für eine Embolie als Modus der Entodermablösung besonders ungünstig. Auch wenn die Bildung dotterhaltiger Zellfragmente im Innern der Embryonen bei der Beobachtung von Lebendmaterial zunächst eine Delamination vortäuschen kann (vgl. Gemmill, 1922), liegt bei Anemonia sulcata die Invaginationsgastrula (Abb. 24) vor. Diesbezüglich zeigen die Gastrulae anderer Actiniaria mit mäßig dotterhaltigen Oocyten (vgl. Appellöf, 1900; Cary, 1910; Nyholm, 1943) zwar prinzipielle Gemeinsamkeiten mit Anemonia, wobei aber Eigenschaften wie der außergewöhnliche Dotterreichtum den Prozeß der Invagination, wie oben näher erläutert, noch mehr modifiziert.

Die zum Aufbau der zweischichtigen Körperwand erforderliche Annäherung von Ektoderm und invaginierendem Entoderm kann nur bewerkstelligt werden, wenn die in dem zu verdrängenden Blastocoel liegenden Speicherstoffe ins Gastrocoel gelangen. Somit steht die Frage nach dem Modus, der diesen Transfer ermöglicht, im Mittelpunkt der Diskussion. Die vorliegenden Befunde legen nahe, daß dotterhaltige Zellteile vorwiegend durch größere Lücken zwischen den Entodermzellen ins Gastrocoel übertreten (Abb. 27). Darüber hinaus bilden die Zellen der Blastoporusregion Fortsätze aus, die die Nährsubstanzen teilweise umhüllen (Abb. 21, 23). Diese Beobachtungen lassen 
prinzipiell zwei Interpretationsmöglichkeiten zu. Entweder werden gerade Speicherstoffe phagocytiert und ins Entoderm eingelagert, oder diese Befunde sind als Indiz für die Endphase einer "Filtration des Nahrungsdotters" (Korschelt \& Heider, 1909) zu werten, wobei die Entodermzellen zunächst Nährsubstanzen aus dem Blastocoel resorbieren und dann wieder ins Gastrocoel entlassen. Dieses Phänomen bleibt offensichtlich aber auf die Blastoporusregion beschränkt. Nach dem gegenwärtigen Stand der Untersuchungen kann davon ausgegangen werden, daß, wenn tatsächlich eine "Filtration des Nahrungsdotters" vorliegt, ihr nur untergeordnete Bedeutung zukommt.

Die Speicherstoffe verbleiben, wie lichtmikroskopisch auch bei anderen ActiniariaLarven gezeigt wurde (Nyholm, 1943; Schäfer, 1981), noch längere Zeit im Gastrocoel, was auf eine vorwiegend lecithotrophe Ernährung der Larven hindeutet. Der Blastoporus verengt sich mit fortschreitender Entwicklung, ohne aber zu verwachsen. Der von den Zellen der Blastoporusregion gebildete Mikrovillisaum (Abb. 23, 28) übernimmt eine Art Siebfunktion und verhindert das Austreten des Nährmaterials ins umgebende Medium, bevor es der Resorption und endgültig seiner Verwertung anheimfällt. Larkman \& Carter (1984) beschreiben, daß Ektodermzellen der Gastrulae von Actinia fragacea Spermien, die sich noch im Wasser befinden, phagocytieren. Ein ähnliches Verhalten war bei Anemona-Gastrulae nicht festzustellen.

\section{Zooxanthellen}

Zooxanthellen sind nicht nur Symbionten riffbildender Scleractinia, auch bei anderen Anthozoa sind sie, vorwiegend im Entoderm, häufig anzutreffen. Bei Actiniaria wurde inzwischen nachgewiesen, daß sie deren Energiehaushalt positiv beeinflussen (Sebens, 1980). Die Oocyten von Anemonia enthalten zahlreiche Zooxanthellen, die aber teilweise vor allem während der Frühentwicklung bis zur Blastula abgestoßen werden. Die vorliegenden Befunde legen nahe, daß Zusammenhänge zwischen dem Ablauf der Ontogenese und Zooxanthellenverlust der Embryonen bestehen. Zunächst werden unmittelbar unter der Oberfläche liegende Symbionten während der heftigen Corticalreaktion zusammen mit anderen Plasmateilen abgesprengt (Schäfer, 1984). Weiterhin dürfte der Zerfall des Deutoplasmas ein wesentlicher Grund sein, warum viele Zooxanthellen aus ihrem Verband mit anderen Zellen bzw. Zellteilen isoliert werden.

Die Beziehung zwischen symbiontischen Algen und Cnidaria ist nach wie vor Gegenstand zahlreicher Untersuchungen (vgl. Muscatine, 1974). Dabei steht $u$. a. die Problematik im Vordergrund, ab wann, wie und ob überhaupt eine Infektion von Larven und Jungtieren mit Zooxanthellen stattfindet. Hierzu legen die Beobachtungen an den Embryonen von Anemonia zusammenfassend folgende Rückschlüsse nahe: Zooxanthellen werden über die Oocyten auf den Keim übertragen. Auch wenn der Keim seinen Bestand an Symbionten drastisch verringert, muß davon ausgegangen werden, daß die in Jungtieren beobachteten Zooxanthellen auf diejenigen zurückgehen, die vom Entoderm des Muttertieres an die Oocyten (vgl. Schäfer, 1984) und damit an die Embryonen weitergegeben werden. 


\section{SCHLUSSBETRACHTUNG}

Bei Anemonia sulcata ist der gesamte Ablauf der Ontogenese bis zur späten Gastrula durch den Dotterreichtum der Oocyten geprägt. Besonders auffällig ist die extrem verzögerte Sonderung der Blastomeren. Deutlich treten sie erst im Stadium der Blastula in Erscheinung, wenn sich das Blastoderm peripher epithelial anordnet und das Innere des Keimes in dotterhaltige Zellfragmente zerfällt. Die Anhäufung der Speicherstoffe im Innern des Keimes verschleiert das Bild der Coeloblastula (Abb. 12-15) und behindert die Invagination des Entoderms, die nur möglich wird, indem die dotterhaltigen Zellteile vom Blastocoel ins Gastrocoel übertreten. Die elektronenoptischen Befunde (Abb. 27) bestätigen im Prinzip die erstmals von Appellöf (1900) geäußerte Vermutung, daß die nährstoffhaltigen Partikel zwischen den Entodermzellen hindurch in den Urdarm gelangen. Aufgrund der Beobachtungen in der Blastoporusregion (Abb. 21, 23) muß auch ein ProzeB in Betracht gezogen werden, der mit einer "Filtration des Nahrungsdotters" (vgl. Korschelt \& Heider, 1909) zu vergleichen ist.

Innerhalb der Anthozoa ist von einigen dotterreichen Octocorallia-Keimen Moruladelamination als Gastrulationsmodus bekannt (vgl. Siewing, 1969; Mergner, 1971). Der ebenfalls dotterreiche Anemonia-Keim hält trotz aller Voraussetzungen, die eine Invagination als umständlich erscheinen lassen, an der klassischen Embolie fest. Dies deutet nach Untersuchung der Ontogenese von Anemonia sulcata letztendlich darauf hin, daß die Invagination als Modus der Entodermablösung tiefer begründet sein muß. Sie ist als, stammesgeschichtlich gesehen, ursprüngliches Merkmal auch bei solchen Anthozoa, deren Oocyten relativ dotterreich sind, genetisch fixiert.

Danksagungen. Herrn Prof. Dr. R. Siewing (Erlangen) gilt mein besonderer Dank für vielfachen Rat bei der Diskussion der Ergebnisse und die stete Förderung meiner Untersuchungen. Weiterhin danke ich Frau Chr. Trommer (Erlangen) für die ausgezeichnete technische Unterstützung in der Elektronenmikroskopie sowie Frau U. Kämpf (Erlangen) für die sorgfältige Ausführung der Zeichenarbeiten. Ebenso danke ich Frl, C. Pomrenke, Frl. E. Schmidt (beide Erlangen) und Frl. E. Schäfer (Heidelberg) für wertvolle technische Hilfe.

\section{LITERATUR}

Appellöf, A., 1900. Studien über Actinien-Entwicklung. - Bergens Mus. Årb. 1, 3-99.

Cary, L. R., 1910. The formation of germ layers in Actinia bermudensis. - Biol. Bull. mar. biol. Lab., Woods Hole 19, 339-346.

Chia, F. S. \& Spaulding, J. G., 1972. Development and juvenile growth of the sea anemone, Tealia crassicornis. - Biol. Bull. mar. biol. Lab., Woods Hole 142, 206-218.

Dewel, W. C. \& Clark, W. H., Jr., 1974. A fine structural investigation of surface specializations and the cortical reaction in the egg of the cnidarian Bunodosoma cavernata. - J. Cell Biol. 60,78-91.

Gemmill, J. F., 1920. The development of the sea anemones, Metridium dianthus (Ellis) and Adamsia palliata (Bohad). - Phil. Trans. R. Soc. (Ser. B) 200, 351-375.

Gemmill, J. F., 1922. The development of the sea anemone Bolocera tuediae (Johnst.), - Q. Jl microsc. Sci. $65,577-587$.

Graeffe, E, 1884. Ubersicht der Seethierfauna des Golfes von Triest nebst Notizen über Vorkommen, Lebensweise, Erscheinungs- und Fortpflanzungszeit der einzelnen Arten, II Coelenteraten. - Arb. zool. Inst. Univ. Wien 5, 333-362.

Korschelt, E. \& Heider, K., 1909. Lehrbuch der vergleichenden Entwicklungsgeschichte der Thiere. Allgemeiner Theil. Fischer, Jena, $896 \mathrm{pp}$.

Lacaze Duthiers, H. de, 1972. Développement des coralliaires I. - Archs Zool exp. gen. 1, 289-396. 
Larkman, A. U. \& Carter, M. A., 1984. The apparent absence of a cortical reaction after fertilization in a sea anemone. - Tissue \& Cell 16, 125-130.

Mergner, H., 1971. Cnidaria. In: Experimental embryology of marine and freshwater invertebrates. Ed. by G. Reverberi. North Holland Publ. Co., London, 1-84.

Muscatine, L., 1974. Endosymbiosis of cnidarians and algae. In: Coelenterate biology. Ed. by L. Muscatine \& H. M. Lenhoff. Acad. Press, New York, 359-395.

Nyholm, K. G., 1943. Zur Entwicklung und Entwicklungsbiologie der Ceriantharien und Aktinien. - Zool. Bidr. Uppsala 22, 87-248.

Pax, F., 1936. Anthozoa. - Tierw. Nord- u. Ostsee 3e2, 81-317.

Schäfer, W, 1981. Fortpflanzung und Sexualität von Cereus pedunculatus und Actinia equina (Anthozoa, Actiniaria). - Helgoländer Meeresunters. 34, 451-461.

Schäfer, W., 1983a. Vergleichende Untersuchungen über Struktur und Genese der Anthozoenoocyten. Teil I: Frühentwicklung und innere Differenzierung der Oocyten. - Zool. Jb. (Anat. Ontogenie Tiere) $109,407-448$.

Schäfer, W., 1983b. Vergleichende Untersuchungen über Struktur und Genese der Anthozoenoocyten. Teil II: Spätere Stadien der Eientwicklung und phylogenetische Schlußfolgerungen. - Zool. $\mathrm{Jb}$. (Anat. Ontogenie Tiere) 110, 343-364.

Schäfer, W., 1984. Fortpflanzung und Entwicklung von Anemonia sulcata (Anthozoa, Actiniaria). I. Fortpflanzungszyklus und Struktur der Oocyten vor und nach der Besamung. - Helgoländer Meeresunters. 38, 135-148.

Schäfer, W. \& Schmidt, H., 1980. The anthozoan egg: Differentiation of internal oocyte structure. In: Developmental and cellular biology of coelenterates. Ed. by P. Tardent \& R. Tardent. Elsevier, Amsterdam, 47-52.

Schmidt, H. \& Schäfer, W., 1980. The anthozoan egg: trophic mechanisms and oocyte surfaces. In: Developmental and cellular biology of coelenterates. Ed. by P. Tardent \& R. Tardent. Elsevier, Amsterdam, 41-46.

Sebens, K. P., 1980. The regulation of asexual reproduction and indeterminate body size in the sea anemone Anthopleura elegantissima (Brandt). - Biol. Bull. mar. biol. Lab., Woods Hole 158, 370-382.

Sembrat, K., 1956. Experimental analysis of the early developmental stages of the sea anemone Sagartia leucolena Verrill. - Zoologica Pol. 7, 129-153.

Siebert, A. E., Jr., 1974. A description of the embryology, larval development, and feeding of the sea anemones Anthopleura elegantissima and A. Xanthogrammica. - Can. J. Zool. 52, 1383-1388.

Siebert, A. E., Jr. \& Spaulding, J. G., 1976. The taxonomy, development and brooding behavior of the anemone Cribrinopsis fernaldi sp. nov. - Biol. Bull. mar. biol. Lab., Woods Hole 150 $128-138$.

Siewing, R., 1969. Lehrbuch der vergleichenden Entwicklungsgeschichte der Tiere. Parey, Hamburg, $531 \mathrm{pp}$.

Spaulding, J. G., 1974. Embryonic and larval development in sea anemones (Anthozoa: Actiniaria). - Am. Zool. 14, 511-520.

Stevenson, T. A., 1935. The British sea anemones. Adlard, London, 2, 1-426.

Wischnitzer, S., 1970. The annulate lamellae. - Int. Rev. Cytol. 27, 65-100. 